\title{
Retiming Sequential Circuits with Multiple Register Classes *
}

\author{
Klaus Eckl Christian Legl \\ Institute of Electronic Design Automation \\ Technical University of Munich \\ 80290 Munich, Germany \\ Klaus.Eckl@ei.tum.de \\ Christian.Legl@ei.tum.de
}

\begin{abstract}
Retiming is an efficient technique for redistributing registers in synchronous circuits in order to improve the circuit performance. However, the traditional retiming approaches cannot handle circuits whose registers are controlled by different clock, reset, and load enable signals. We present basic theory and a comprehensive retiming approach for circuits with multiple clock, reset, and load enable signals. We retime these circuits having multiple register classes without explicitly modeling the reset or the load enable by additional logic. The presented concepts can be combined with a wide range of existing retiming approaches. Experimental results from retiming real designs for clock period minimization show the efficiency of the new approach.
\end{abstract}

\section{Introduction}

In modern VLSI design the optimization of synchronous sequential circuits is a key issue. In order to optimize synchronous circuits, combinational optimization techniques are usually applied in synthesis systems while sequential optimization techniques are only rarely used.

A very promising sequential optimization technique is called retiming. Retiming moves registers across combinational logic blocks without changing the logic function inside the blocks. Leiserson and Saxe [9, 10] showed that retiming can be used for minimizing the clock period as well as for minimizing the number of registers under a clock period constraint. It can be applied to circuits with levelsensitive latches [11] or edge-triggered flip-flops [10, 16]. Several extensions to retiming $[15,2,3]$ as well as efficient implementations $[16,13]$ have been proposed which show that retiming is even applicable to large circuits. In this paper, we focus on sequential circuits having edgetriggered flip-flops as registers. However, the concepts and approaches of this paper can also be transferred to circuits with level-sensitive latches.

Most retiming approaches assume that edge-triggered sequential circuits have only a single clock. Industrial designs, however, are usually multiple-clock systems, i.e., dif-

${ }^{*}$ This work was supported in part by DFG under grant AN 125/17. ferent registers may be connected to different clocks. This fact complicates the retiming problem because additional constraints are imposed on register movements in order to preserve temporality [17]. Lockyear and Ebeling [11], and Ishii et al. [7] already tackled the problem of retiming multiphase level-clocked circuits. However, their approaches are restricted to the special class of well-formed circuits in which the sequence of clock phases has the same order along any path through the circuit. Thus, these approaches need not impose additional constraints on register movements. In general multiple-clock circuits, however, these constraints must be taken into account explicitly.

Most of the existing retiming approaches assume that the circuit has a single reset signal for all registers which is activated only once at the beginning of circuit operation $[19,5,14]$. Thus, they only tackle the problem of computing an initial state for the retimed circuit that is equal to the initial state of the original circuit. Singhal et al. [18] generalized the retimed initial state problem to the retimed reset state problem by allowing, e.g., different reset lines to be activated during different clock cycles. They showed that register moves may be constrained due to the assumption about reset activation and due to different reset signals connected to different registers. However, they only looked at a single retiming move and did not present an overall retiming algorithm that finds an optimal retiming in accordance with the retimed reset state problem.

Registers may have a load enable (also called clock enable) capability. It is desirable that retiming preserves the load enables because modeling a load enable by a simple register with extra logic increases area and delay costs. E.g., many FPGAs offer the load enable capability for free, making its use imperative for good FPGA design. Thus, a retiming approach should be able to move registers together with their load enables across logic blocks. This problem was addressed in [1] and [6]. Camposano and Plöger [1] stated several conditions for a valid retiming step involving load enable registers. However, they did not present an overall approach for computing a retiming solution that satisfies these constraints. A first idea for solving the retiming problem in circuits with multiple load enable and clock signals was introduced in [8], but no practical implementation and no experimental results were shown.

In this paper, we present basic theory and an efficient 
approach for retiming synchronous circuits where different registers can be connected to different clocks, asynchronous and synchronous reset signals, and load enable signals. After some background information on traditional retiming in Section 2, we show in Section 3 how to partition the registers into classes. On the basis of these classes we define a circuit transformation called multiple-class retiming which guarantees that the retimed circuit is a sufficiently old replacement [9] of the original circuit. In Section 4 some theoretical aspects of multiple-class retiming are shown which lead to an efficient implementation.

Section 5 presents an efficient multiple-class retiming approach. It transforms the problem of retiming a multipleclass sequential circuit into a traditional single-class retiming problem. Then, any state-of-the-art retiming approach can be used which has been presented, e.g., for clock period minimization or register minimization under a clock period constraint. Finally, the multiple-class sequential circuit is updated according to the solution for the single-class retiming problem. This also includes the computation of equivalent asynchronous and synchronous reset states for the retimed circuit.

In order to show the applicability, we implemented our new approach and applied it to the clock period minimization problem. Experimental results in Section 6 show the efficiency of our multiple-class retiming approach.

\section{Background}

In this section, we briefly review the terminology and the graph model for single-class sequential circuits.

Leiserson and Saxe $[9,10]$ model a single-class sequential circuit as a vertex-weighted, edge-weighted, directed multigraph $G=\langle V, E, d, w\rangle$, called retiming graph. Each combinational logic block and each input and output of the sequential circuit is represented by a unique vertex $v \in V$. Additionally, a host vertex $v_{\text {host }}$ is introduced to model the environment of the circuit. There exists an edge $e_{u v} \in E$ if an output of the logic block represented by vertex $u$ is connected to an input of the logic block represented by vertex $v$. Moreover, there are edges from $v_{\text {host }}$ to all input vertices and from all output vertices to $v_{\text {host }}$.

The vertex weight $d(v)$ is the propagation delay of the logic block of vertex $v$. The edge weight $w\left(e_{u v}\right)$ indicates the number of registers along the interconnection from vertex $u$ to vertex $v$. A path $p: u \sim v$ in the graph is a sequence of edges from vertex $u$ to vertex $v$. The path weight $w(p)$ is the sum of the edge weights along the path.

A retiming is an integer-valued vertex labeling $r: V \rightarrow Z$. A retiming (or lag) value $r(v)$ denotes the number of registers that are moved from the outputs to the inputs of $v$. The retimed graph $G_{r}=\left\langle V, E, d, w_{r}\right\rangle$ can be derived from the original graph $G$ by computing the retimed edge weights,

$$
w_{r}\left(e_{u v}\right)=w\left(e_{u v}\right)+r(v)-r(u) .
$$

Similarly, the retimed path weight $w_{r}(p)$ of a path $p: u \leadsto v$ is computed by $w_{r}(p)=w(p)+r(v)-r(u)$. A retiming $r: V \rightarrow Z$ is legal [10] if for each edge $e_{u v}$ the retimed edge weight $w_{r}\left(e_{u v}\right)$ is nonnegative, i.e., $w_{r}\left(e_{u v}\right) \geq 0$.
A retiming $r^{\prime}: V \rightarrow Z$ with $r^{\prime}\left(v_{\text {host }}\right) \neq 0$ moves registers across inputs and outputs. However, an equivalent retiming $r$ for which $r\left(v_{\text {host }}\right)=0$ holds can always be obtained from $r^{\prime}$ by the normalization [5]

$$
r(v)=r^{\prime}(v)-r^{\prime}\left(v_{\text {host }}\right) \text {. }
$$

In the sequel we will always assume that $r\left(v_{\text {host }}\right)=0$ holds.

\section{Multiple-class retiming}

Before looking at the problem of multiple class retiming, we present the types of registers we are going to support and how to model these different types in a retiming graph.

The left hand side of Fig. 1 shows a generic register $l$ between some gates $u$ and $v$. Each register $l$ is at least connected to a data input and output signal and a clock signal $c l k$. A register may be connected to further control signals which are

- an asynchronous reset signal ars causing an asynchronous set or clear behavior,

- a synchronous reset signal srs causing a synchronous set or clear behavior,

- a load enable (or clock enable) signal le.

Contrary to the circuits handled by most of the existing retiming approaches we now also consider circuits containing multiple types or classes of registers.

Definition 1 (Register class) A class $C$ of registers is characterized by a tuple (clk, le, ars, srs) of signals. A register $l$ is contained in class $C$ iff each of its control signals is identical to the corresponding signal in the tuple of class $C$.

The case that some register does not use, e.g., a load enable is modeled by connecting a constant 0 signal to the input $l e$. Two clock signals are identical if they have the same period and phase offset. Two load enable or reset signals are identical if the signals are logical equivalent. The (a)synchronous reset state is determined by the reset signal being connected to either a set or clear input of the register. However, the class of a register does not depend on the reset state itself. Registers that belong to the same class are denoted compatible registers.

In the sequel, a circuit containing different classes of registers is called a multiple-class circuit in contrast to a singleclass circuit which contains only one class of registers. For a multiple-class circuit it is no longer sufficient to store the number of registers $w\left(e_{u v}\right)$ on an edge $e_{u v}$ of the retiming graph, as each register may belong to a different class. This information needs to be modeled in the retiming graph.

Therefore, we introduce a modified retiming graph $G^{m c}=\langle V, E, d, \underline{l}\rangle$ which we call mc-retiming graph or short $m c$-graph (an example is depicted right hand side of Fig. 1). Instead of the edge values $w\left(e_{u v}\right)$ we place an ordered list $\underline{l}\left(e_{u v}\right)=\left[l_{1}, \ldots, l_{w\left(e_{u v}\right)}\right]$ on each edge. This list indicates a sequence of $w\left(e_{u v}\right)$ registers on this edge. $l_{1}$ corresponds to the register closest to the source of the edge, while $l_{w\left(e_{u v}\right)}$ is the register closest to the sink of the edge. An optional 


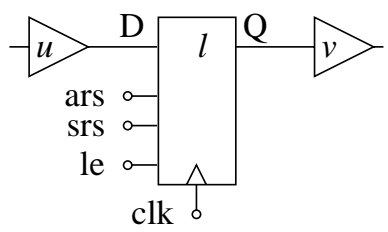

circuit

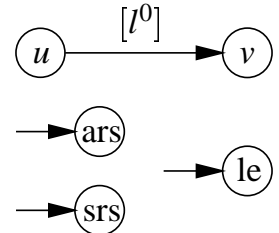

$\Longrightarrow$ mc-retiming graph $G^{m c}$
Figure 1. Modeling registers in a mc-retiming graph.

superscript $j$ at some register $l_{i}^{j}$ denotes the index of the corresponding register class $C_{j}$. Additionally, for each load enable and reset signal we introduce an output vertex in the mc-retiming graph.

For an mc-graph we keep the notion of edge weights which are now given by the length of the lists: $w\left(e_{u v}\right)=$ $\left|\underline{l}\left(e_{u v}\right)\right|$. Similarly to the single-class retiming graph (scgraph) we apply the concept of register lists also to paths: The sequence of registers on path $p: u \leadsto v$ is given by the list $\underline{l}(p)$ and $l_{i}(p)$ denotes the $i$-th register on that path.

A basic mc-retiming step for a vertex $v$ can now be performed as depicted in Fig. 2. E.g., for one forward mcretiming step at vertex $v$, there must be a complete layer of registers at the incoming edges of $v$ which comprises the last registers in each list. During the forward retiming step, the registers of the layer are removed from their list, and a new layer is inserted at the fanout edges of $v$ by prepending a register to the list of each outgoing edge.

Of course, for multiple-class circuits certain restrictions apply to the validity of such a mc-retiming step. A mcretiming step is valid if it yields a circuit which is a sufficiently old replacement [9] of the original circuit. It has been shown in [1] for load enable signals that such a retiming step is valid if all registers of the layer to be moved are connected to the same load enable signal. The same condition applies to the clock signal in order to preserve the temporal equivalence of the circuit [17]. Registers with reset signals can be moved if the corresponding signals are identical [18]. Since our definition of register classes is based on the same conditions, we can relate the validity of a mcretiming step to the classes of the registers to be moved:

\section{Theorem 1 (Validity of a mc-retiming step)}

A mc-retiming step as shown in Fig. 2 is valid if all registers to be moved belong to the same class.

The proof of this theorem directly follows from $[17,1$, 18]. Note that by examining the state space or the logic functions we may find few cases where registers of different classes can be moved across a vertex while still preserving circuit equivalence. However, such extensive analysis would deteriorate the efficiency of retiming. Therefore, we do not consider such cases.

As in traditional single-class retiming, we can also define a retiming for a multiple-class circuit as an integer valued vertex labeling $r: V \rightarrow Z$. The validity of such a retiming for a multiple-class circuit is given by the following theorem.

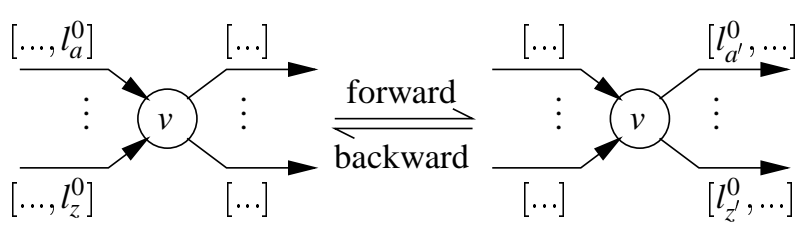

Figure 2. Performing a basic mc-retiming step

Theorem 2 (Legal mc-retiming) A retiming $r: V \rightarrow Z$ generating a circuit which is a sufficiently old replacement of the original circuit is a legal retiming for a multiple-class circuit, short a legal mc-retiming, if it can be implemented by a sequence of valid mc-retiming steps.

The proof of this theorem follows from the validity of each single basic mc-retiming step. In the following sections we will show how a legal mc-retiming can be efficiently computed.

\section{Theory of multiple-class retiming}

We will now derive some properties of mc-retiming and we show its relation to sc-retiming. This relation will lead to an efficient implementation of mc-retiming. The proofs of the theorems can be found in [4].

Let a legal retiming $r: V \rightarrow Z$ be given for a scretiming problem where no register is moved across the inputs/outputs of the circuit, i.e., $r\left(v_{\text {host }}\right)=0$. It was shown in [12] that the retiming values are bounded from above and from below as given by the following theorem.

Theorem 3 For each vertex $v$ of $G$, let $p_{I}(v)$ and $p_{O}(v)$ be minimum weighted paths from the host vertex $v_{\text {host }}$ to $v$ and from $v$ to $v_{\text {host }}$, respectively. Then, any legal sc-retiming $r: V \rightarrow Z$, with $r\left(v_{\text {host }}\right)=0$, satisfies

$$
r_{\min }(v) \leq r(v) \leq r_{\max }(v),
$$

where $r_{\min }(v)=-w\left(p_{I}(v)\right)$, and $r_{\max }(v)=w\left(p_{O}(v)\right)$.

Intuitively, we can not move more registers forward or backward across $v$ than the minimum number of registers available on any path from $v_{\text {host }}$ to $v$ or from $v$ to $v_{\text {host }}$, respectively. Theorem 3 guarantees for sc-retiming that, e.g., up to $r_{\text {max }}(v)$ registers can be moved backward across $v$. However, for mc-retiming this may not be possible since different register classes can prohibit some retiming steps. This is illustrated by the following example.

Figure 3 shows a part of a mc-retiming graph. For vertex $v_{1}$ a minimum weighted path to the host vertex is given by $p_{O}\left(v_{1}\right)=\left(v_{1}, v_{3}, v_{\text {host }}\right)$. It carries the register list $\underline{l}\left(p_{O}\right)=\left[l_{a}^{0}, l_{b}^{0}\right]$ and has weight $w\left(p_{O}\right)=2$. Thus, for scretiming, $r_{\max }\left(v_{1}\right)=w\left(p_{O}\right)=2$ registers could be moved backward across $v_{1}$. For mc-retiming, the first backward mc-retiming step would be valid because the corresponding layer is comprised by compatible registers $l_{a}^{0}$ and $l_{c}^{0}$. For the second backward move at $v_{1}$ we first move register $l_{d}^{1}$ across vertex $v_{2}$ which is a valid step. However, now the registers $l_{b}^{0}$ and $l_{d}^{1}$ at the fanout edges of $v_{1}$ belong to different classes $C_{0}$ and $C_{1}$ which prohibits any further backward move. 


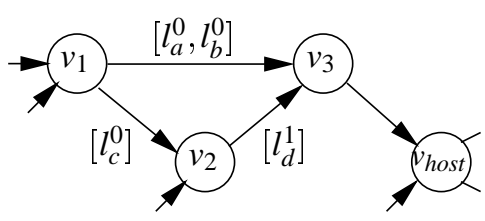

Figure 3. Part of a mc-retiming graph $G^{m c}$.

We will now investigate the condition under which a retiming value is a valid mc-retiming value.

Definition 2 (Valid mc-retiming value) A retiming value $r(v)$ is a valid $m c$-retiming value iff $r(v)$ can be implemented by a sequence of valid mc-retiming steps.

The validity of $r(v)$ is directly related to the compatibility of the registers in the layers that can be reached on the incoming and outgoing paths of vertex $v$.

Theorem 4 (Conditions for a valid mc-retiming value) A retiming value $r(v)>0$ is a valid $m c$-retiming value iff

a) $r(v) \leq r_{\max }(v)$,

b) $r(v)-1$ is a valid mc-retiming value, and

c) all registers $l_{r(v)}(p)$ on all paths starting at vertex $v$ are compatible.

A retiming value $r(v)<0$ is a valid $m c$-retiming value iff

a) $r(v) \geq r_{\min }(v)$,

b) $r(v)+1$ is a valid mc-retiming value, and

c) all registers $l_{w(p)+1+r(v)}(p)$ on all paths ending at vertex $v$ are compatible.

Trivially, $r(v)=0$ is always a valid mc-retiming value.

From Theorem 4 it follows that there exist a unique lower and upper bound for the valid mc-retiming values of each vertex which we will denote forward mc-retiming bound $r_{\text {min }}^{m c}(v)$ and backward mc-retiming bound $r_{\max }^{m c}(v)$, respectively. Because of Theorem 4 these bounds never exceed the sc-retiming bounds, i.e.,

$$
r_{\text {min }}(v) \leq r_{\text {min }}^{m c}(v) \leq r(v) \leq r_{\text {max }}^{m c}(v) \leq r_{\max }(v) .
$$

By Theorem 3, a legal sc-retiming only guarantees that $r_{\min }(v) \leq r(v) \leq r_{\max }(v)$. If we also want to guarantee a legal mc-retiming solution, we must additionally incorporate the mc-retiming bounds (4) into the mc-retiming problem formulation. This leads to the following pivotal theorem which states the conditions for a legal mc-retiming solution.

Theorem 5 (Legal mc-retiming) A retiming $r: V \rightarrow Z$ is a legal mc-retiming iff the following conditions hold:

$$
\begin{aligned}
\forall e_{u v} \in E \quad: \quad r(u)-r(v) \leq w\left(e_{u v}\right) \\
\quad r\left(v_{\text {host }}\right)=0 \\
\forall v \in V \quad: \quad r_{\min }^{m c}(v) \leq r(v) \leq r_{\max }^{m c}(v) .
\end{aligned}
$$

Conditions (5) and (6) are the same as for sc-retiming and simply reflect the demand for positive retimed edge weights and prohibition of input/output moves, respectively. Additionally, condition (7) allows only valid mc-retiming values. These values can be implemented by valid mcretiming steps. Thus, $r$ represents a legal mc-retiming according to Theorem 2 .
Corollary 1 A mc-retiming $r: V \rightarrow Z$ is legal iff $r$ is a legal sc-retiming and all retiming values fulfill the constraints given by the mc-retiming bounds.

Corollary 1 directly leads to an efficient approach for mc-retiming. We can use any sc-retiming approach during the search for an optimal mc-retiming solution as long as we satisfy the additional mc-retiming bounds for the retiming values. The following section will show that these mc-retiming bounds can be incorporated into the traditional sc-retiming problem formulation in a natural way.

\section{Efficient algorithm for mc-retiming}

In the last section we showed that a mc-retiming solution can be obtained by performing sc-retiming with additional constraints on the retiming values. In order to do so we now propose an efficient mc-retiming approach which performs the following three steps:

1. Compute backward and forward mc-retiming bounds.

2. Compute sc-retiming which fulfills the mc-retiming constraints using any generic retiming approach.

3. Move the registers in the graph $G^{m c}$ to their final positions according to retiming values obtained in step 2 .

The following subsections will explain these steps in more detail.

\subsection{Computing the mc-retiming constraints}

We will only show how to compute the backward mcretiming bounds $r_{\max }^{m c}(v)$. All results derived for this case can be easily transfered to the computation of the forward mc-retiming bounds $r_{\text {min }}^{m c}(v)$.

From Section 4 we know that a unique value $r_{\text {max }}^{m c}$ exists for each vertex of the graph $G^{m c}$. We call a retiming $r^{*}$ where $r^{*}(v)=r_{\max }^{m c}(v)$ for each vertex $v$ a maximal backward retiming because it moves the registers as far as possible backward toward the primary inputs. We can compute a maximal backward retiming, and thus the values $r_{\text {max }}^{m c}$, by applying valid backward mc-retiming steps as long as possible while counting the number of registers which move across each vertex. The next theorem shows that no matter in which order we apply the basic retiming steps, we always end up with the unique maximal backward retiming $r^{*}$.

Theorem 6 (Maximal backward retiming) Let $r$ be a legal mc-retiming which has already been implemented by valid mc-retiming steps. Then, as long as there exists a vertex $u$ with $r(u)<r_{\max }^{m c}(u)$, there exists at least one vertex $v$ for which a valid mc-retiming step can be applied.

Proof : Let $u$ be a vertex with $r(u)<r_{\max }^{m c}(u)$. Then, each outgoing path $p: u \leadsto v_{\text {host }}$ has a register $l_{1}(p)$ on it, and all these registers are compatible. By an iterative procedure [4], we can show that either $u$ or some vertex $v$ in the transitive fanout of $u$ is retimeable by a valid mc-retiming step. 


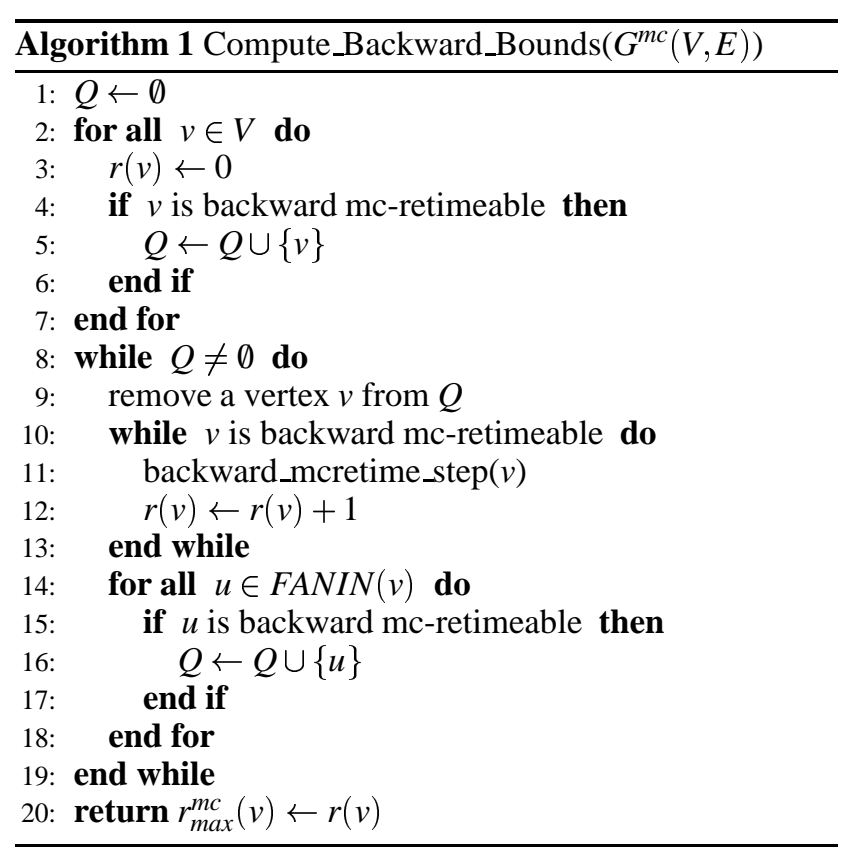

Algorithm 1 shows an efficient procedure which computes the backward mc-retiming bounds $r_{\text {max }}^{m c}$. First, all vertices that are amenable for a backward mc-retiming step are added to the set $Q$. In the main loop one vertex $v$ is removed from $Q$ at a time and retimed backward as often as possible while increasing its retiming value accordingly. Then, each fanin vertex $u$ of $v$ which became backward retimeable due to the retiming of $v$ is inserted into the set $Q$. The loop terminates when the set $Q$ is empty. Then, each retiming value is equal to the backward mc-retiming bound $r_{\max }^{m c}$.

The runtime of this algorithm is $O(k(V+E))$ where $k=$ $\max \left\{r_{\max }^{m c}(u) \mid u \in V\right\}$. This runtime complexity is strictly less than the complexity of existing sc-retiming approaches. E.g., the FEAS algorithm used for minimum clock period retiming takes a runtime of $O(V E \cdot \lg V)$ [10]. Algorithms for register minimization under a clock period constraint have even higher runtime complexities. Thus, the computation of the mc-retiming bounds has only a small impact on the runtime of the overall mc-retiming approach. This will also be shown by the results in Section 6 .

\subsection{SC-Retiming under mc-retiming constraints}

The sc-retiming problem is an integer linear program (ILP) with difference constraints only. Thus, it can be solved efficiently, e.g., by shortest-path algorithms for minimum period retiming and by minimum-cost flow algorithms for register minimization [10].

Corollary 1 shows that we can generate a legal mcretiming by searching for a legal sc-retiming with additional retiming constraints imposed. Using $r\left(v_{\text {host }}\right)=0$, we can rewrite the mc-retiming constraint (7) using two difference constraints,

$$
\begin{aligned}
& r\left(v_{\text {host }}\right)-r(v) \leq-r_{\min }^{m c}(v) \\
& r(v)-r\left(v_{\text {host }}\right) \leq r_{\max }^{m c}(v)
\end{aligned}
$$
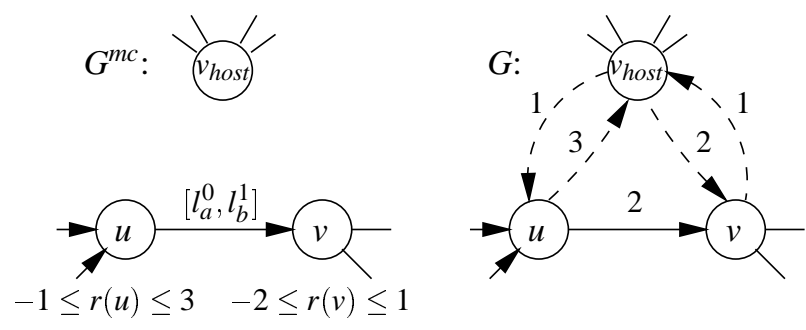

Figure 4. Transforming mc-graph to sc-graph.

If we add these constraints to the sc-retiming problem, we again have an ILP with difference constraints only. Thus, we can still use the same efficient sc-retiming approaches but now we guarantee that the mc-retiming bounds are fulfilled. Note that only the number of constraints increases but not the number of variables. In fact, in the Minaret approach [13] it was shown that additional constraints may be used to reduce the size of the ILP such that less time is needed to solve the problem.

Other approaches, like the FEAS algorithm [10] or Reverse Retiming [5], use the retiming graph as the basis for their computation. By the following steps, we can transform the mc-graph $G^{m c}=\langle V, E, d, \underline{l}\rangle$ into a sc-graph $G=$ $\left\langle V, E \cup E_{C}, d, w\right\rangle$ such that the mc-retiming constraints for $G^{m c}$ are inherently modeled in the sc-graph $G$.

1. Each edge $e_{u v}$ in $G^{m c}$ is translated into an edge $e_{u v}$ in $G$ with the register list $\underline{l}\left(e_{u v}\right)$ substituted by the weight $w\left(e_{u v}\right)=\left|\underline{l}\left(e_{u v}\right)\right|$.

2. We model the constraints (8) and (9) by an additional set $E_{C}$ of constraint edges. For each vertex $v$ we introduce an edge from the host vertex to vertex $v$ with $w\left(e_{v_{\text {host }}, v}\right)=$ $-r_{\min }^{m c}(v)$ and an edge from the vertex $v$ to the host vertex with $w\left(e_{v, v_{\text {host }}}\right)=r_{\max }^{m c}(v)$.

The constraint edges enforce that any sc-retiming solution will not move more registers across a vertex $v$ than given by the corresponding constraint edge weight. For example, for the sc-graph $G$ in Fig. 4 it is guaranteed for vertex $u$ that not more than 1 register is moved forward and not more than 3 registers are moved backward. Thus, the given mc-retiming constraints for vertex $u$ will be fulfilled.

The number of constraint edges is only $O(V)$. As the runtime of retiming algorithms is usually quadratic or cubic in the number of vertices, the overall runtime is nearly not affected by the constraint edges. In fact, as proposed in $[16,13]$ a closer look often reveals that many constraint edges are redundant and can be removed because they are dominated by neighboring constraints.

\subsection{Updating the mc-retiming graph}

The last step of our mc-retiming approach is to move the registers of the mc-graph $G^{m c}$ to their final position according to the retiming values. Two problems must be solved. First, a proper sequence of mc-retiming steps must be found. Second, the asynchronous as well as the synchronous reset state must be computed for each moved register. In order to solve both problems, we use a modi- 
fied version of the Update_Registers algorithm proposed by Even et al. [5]. When moving a register backward or forward, the new reset states are computed by backward justification or forward implication, respectively.

It may happen that a backward move across a vertex $v$ is not possible because backward justification fails. Similar to [5] we handle this conflict by decreasing the backward mcretiming bound $r_{\max }^{m c}(v)$ such that the conflicting backward move is no longer allowed. Then, a new retiming solution is computed that prohibits the non-justifiable backward move.

Alternatively, like in [14] we could derive additional retiming constraints already when computing the mc-retiming bounds such that the existence of a backward justification is guaranteed. As noted in [14], this generally results in multiple sets of constraints for which multiple ILPs must be solved. Furthermore, we would have to justify a lot of backward moves which are eventually not implemented in the final retiming solution. Because of this large computational overhead, we dropped this alternative.

\section{Results}

In order to show the effectiveness and efficiency of our approach, we chose clock period minimization as the retiming optimization goal. For ease of implementation, we used the unit delay model to estimate gate delays. Of course, using a more realistic delay model is only a matter of having access to an appropriate timing analyzer. For the generic scretiming approach, we selected Reverse_Retiming [5]. It has the same complexity as FEAS [10] but on average it generates retiming solutions for which the reset state computation is easier [5]. Additionally, we used acceleration techniques for minimum period retiming as proposed in [16].

In order to solve the problems of backward justification and forward implication during reset state computation we use a straight-forward approach based on BDDs. The overall approach for updating the mc-retiming graph is implemented as described in Section 5.3.

We applied our mc-retiming approach to a set of midsized and large benchmark circuits from the LGSynth93 ${ }^{1}$ benchmark suite as well as to a set of industrial designs. Although the LGSynth93 benchmark circuits are single-class circuits, they were selected to show the efficiency of the computation of the mc-retiming bounds. The industrial circuits, denoted ind1 to ind9, are multiple-class circuits.

Table 1 shows characteristic data for the computation of the mc-retiming bounds. The circuit name, the number of gates, and the number of registers are shown in columns 1 to 3 . The number of register classes is given in column \#C. Column \#back and \#forw show the overall number of backward and forward moves that are performed by the maximal backward and forward retiming algorithm, respectively. The last column shows the CPU time (DEC Alpha $41005 / 300$ ) for computing the mc-retiming bounds.

For the industrial examples, the number of classes ranges from 2 to 18. CPU times for the computation of the mc-

\footnotetext{
${ }^{1}$ As in [5], the initial state of each register is assumed to be zero.
}

Table 1. Computation of mc-retiming bounds

\begin{tabular}{|l||r|r||r|r|r||r|}
\hline circuit & \#gate & \#reg & \#C & \#back & \#forw & CPU(s) \\
\hline gcd & 869 & 59 & 1 & 5618 & 621 & 0.63 \\
mm9b & 574 & 26 & 1 & 108 & 29 & 0.10 \\
mult16a & 212 & 16 & 1 & 1747 & 16 & 0.23 \\
s344 & 217 & 15 & 1 & 219 & 164 & 0.07 \\
s382 & 232 & 21 & 1 & 414 & 145 & 0.08 \\
s400 & 246 & 21 & 1 & 448 & 157 & 0.10 \\
s444 & 274 & 21 & 1 & 532 & 191 & 0.10 \\
s526 & 277 & 21 & 1 & 529 & 221 & 0.12 \\
s838.1 & 530 & 32 & 1 & 222 & 98 & 0.12 \\
s953 & 621 & 29 & 1 & 770 & 108 & 0.18 \\
s1238 & 690 & 18 & 1 & 121 & 9 & 0.12 \\
s1423 & 796 & 74 & 1 & 657 & 175 & 0.18 \\
s5378 & 2742 & 163 & 1 & 987 & 2412 & 0.70 \\
s9234.1 & 2796 & 135 & 1 & 3793 & 2448 & 0.92 \\
s38417 & 22520 & 1465 & 1 & 94499 & 40164 & 15.83 \\
ind1 & 1426 & 318 & 18 & 510 & 122 & 0.43 \\
ind2 & 1943 & 103 & 7 & 825 & 62 & 0.50 \\
ind3 & 208 & 46 & 4 & 170 & 111 & 0.07 \\
ind4 & 78 & 7 & 2 & 135 & 20 & 0.03 \\
ind5 & 651 & 38 & 2 & 622 & 120 & 0.20 \\
ind6 & 509 & 33 & 2 & 397 & 10422 & 1.45 \\
ind7 & 635 & 18 & 2 & 595 & 151 & 0.20 \\
ind8 & 311 & 58 & 3 & 129 & 36497 & 4.02 \\
ind9 & 648 & 26 & 2 & 609 & 16067 & 1.62 \\
\hline
\end{tabular}

retiming bounds indicate that maximal backward and forward retiming can be performed very fast. E.g., it takes only 15.83 seconds for circuit s38417 with 22520 gates and 1465 registers.

The minimum period retiming results for the circuits of Table 1 are shown in Table 2. The initial clock period, the initial number of registers, the clock period after retiming, and the number of registers after retiming are shown in the columns denoted initial CP, initial \#reg, final CP, and final \#reg, respectively. Column \#steps shows the actual number of performed mc-retiming steps. Columns titled MINP and $U R E G$ give the CPU times for Reverse_Retiming and updating the mc-retiming graph, respectively.

Table 2 shows a substantial reduction in the clock period for the benchmark circuits as well as for the industrial designs. This indicates that retiming is a very powerful optimization technique also for multiple-class circuits. All backward moves could be justified by our BDD-based approach such that no backtrack was necessary. Comparing CPU times it is important to notice that the impact of computing the mc-retiming bounds on the efficiency of the overall retiming approach is low. This holds even if very efficient acceleration techniques [16] are used for the scretiming algorithm. This stresses the theoretical results of Section 5.1. Please note that the large CPU time in column $U R E G$ is due to the BDD-based reset state computation, which we have implemented in a straight-forward way only.

\section{Conclusion}

In this paper we have presented basic theory and an approach to retiming synchronous circuits where different registers can be connected to different clock, asynchronous and synchronous reset, and load enable signals. In order to manage this variety of registers, we introduced the concept of 
Table 2. Results for clock period minimization

\begin{tabular}{|l||r|r||r|r|r||r|r|}
\hline \multicolumn{1}{|l||}{ circuit } & \multicolumn{2}{c|}{ initial } & \multicolumn{3}{|c||}{ final } & \multicolumn{2}{c|}{ CPU(s) } \\
\cline { 2 - 7 } CP & \#reg & CP & \#reg & \#steps & MINP & UREG \\
mm9b & 32 & 59 & 28 & 77 & 40 & 0.2 & 0.6 \\
mult16a & 30 & 26 & 58 & 27 & 19 & 0.1 & 0.0 \\
s344 & 28 & 16 & 8 & 84 & 491 & 0.1 & 44.8 \\
s382 & 17 & 21 & 19 & 25 & 25 & 0.0 & 0.0 \\
s400 & 17 & 21 & 10 & 48 & 111 & 0.4 & 2.8 \\
s444 & 20 & 21 & 11 & 45 & 153 & 0.9 & 2.6 \\
s526 & 14 & 21 & 9 & 84 & 146 & 0.6 & 3.6 \\
s838.1 & 21 & 32 & 20 & 34 & 2 & 0.1 & 8.2 \\
s953 & 27 & 29 & 22 & 34 & 7 & 0.1 & 0.1 \\
s1238 & 31 & 18 & 30 & 82 & 121 & 0.1 & 1.9 \\
s1423 & 66 & 74 & 59 & 160 & 513 & 0.2 & 170.7 \\
s5378 & 32 & 163 & 27 & 185 & 63 & 0.6 & 0.3 \\
s9234.1 & 55 & 135 & 51 & 135 & 32 & 0.6 & 0.3 \\
s38417 & 65 & 1465 & 45 & 1629 & 952 & 6.6 & 289.5 \\
ind1 & 11 & 318 & 10 & 322 & 4 & 0.4 & 6.9 \\
ind2 & 19 & 103 & 14 & 277 & 687 & 0.7 & 3289.5 \\
ind3 & 7 & 46 & 6 & 45 & 98 & 0.0 & 0.1 \\
ind4 & 6 & 7 & 3 & 38 & 36 & 0.0 & 0.1 \\
ind5 & 11 & 38 & 8 & 181 & 387 & 0.1 & 0.3 \\
ind6 & 8 & 33 & 7 & 83 & 160 & 0.1 & 0.1 \\
ind7 & 13 & 18 & 8 & 188 & 380 & 0.1 & 42.5 \\
ind8 & 8 & 58 & 7 & 94 & 64 & 0.1 & 13.8 \\
ind9 & 10 & 26 & 9 & 105 & 331 & 0.1 & 178.0 \\
\hline
\end{tabular}

register classes. We defined a circuit transformation called mc-retiming which guarantees that the retimed circuit is a valid replacement of the original circuit. Moreover, no additional logic has to be added to the circuit in order to implement the transformation.

Our retiming approach is based on transforming the mcretiming problem into the traditional retiming problem as introduced by Leiserson et al.. Thus, efficient state-of-theart retiming algorithms can be applied for the mc-retiming problem. Experimental results show the efficiency of the presented mc-retiming approach. Being able to retime sequential circuits with multiple register classes will significantly increase the applicability of retiming to real designs.

Based on the proposed mc-retiming approach we are now working on extending our approach in order to handle timing constraints due to multiple-clock systems. A first concept has been presented in [8].

\section{Acknowledgment}

We are very grateful to Dr. Peter Vanbekbergen, Dr. Jean Christophe Madre, Dr. Peter Zepter, and Dr. Albert Wang for many valuable discussions. We also thank Synopsys Inc. for the opportunity of a summer internship. Finally, we highly appreciate the continuous support and steady interest of Prof. Kurt J. Antreich in our work.

\section{References}

[1] R. Camposano and P. G. Plöger. Retiming and highlevel synthesis. In International Workshop on High-LevelSynthesis, pages 191-201, Nov. 1992.

[2] G. De Micheli. Synchronous logic synthesis: Algorithms for cycle-time minimization. IEEE Transactions on ComputerAided Design of Integrated Circuits and Systems, 10(1):6373, Jan. 1991.
[3] S. Dey, M. Potkonjak, and S. G. Rothweiler. Performance optimization of sequential circuits by eliminating retiming bottlenecks. In IEEE/ACM International Conference on Computer-Aided Design (ICCAD), pages 504-509, Nov. 1992.

[4] K. Eckl and C. Legl. A new retiming approach for sequential circuits with multiple flip-flop classes. Technical Report TUM-LRE-98-1, Institute of Electronic Design Automation, Technical University of Munich, Apr. 1998.

[5] G. Even, I. Y. Spillinger, and L. Stok. Retiming revisited and reversed. IEEE Transactions on Computer-Aided Design of Integrated Circuits and Systems, 15(3):348-357, Mar. 1996.

[6] A. T. Ishii. Retiming gated-clocks and precharged circuit structures. In IEEE/ACM International Conference on Computer-Aided Design (ICCAD), pages 300-307, Nov. 1993.

[7] A. T. Ishii, C. E. Leiserson, and M. C. Papaefthymiou. Optimizing two-phase, level-clocked circuitry. In T. Knight and J. Savage, editors, Advanced Research in VLSI and Parallel Systems: Proceedings of the 1992 Brown/MIT Conference, pages 245-264. MIT Press, 1992.

[8] C. Legl, P. Vanbekbergen, and A. Wang. Retiming of edgetriggered circuits with multiple clocks and load enables. In International Workshop on Logic Synthesis (IWLS), volume 1, May 1997.

[9] C. E. Leiserson and J. B. Saxe. Optimizing synchronous systems. Journal of VLSI and Computer Systems, 1(1):4167, Spring 1983.

[10] C. E. Leiserson and J. B. Saxe. Retiming synchronous circuitry. Algorithmica, 6(1):5-35, 1991.

[11] B. Lockyear and C. Ebeling. Optimal retiming of multiphase, level-clocked circuits. In T. Knight and J. Savage, editors, Advanced Research in VLSI and Parallel Systems: Proceedings of the 1992 Brown/MIT Conference, pages 265280. MIT Press, 1992.

[12] B. E. Lockyear. Algorithms for Retiming Level-Clocked Circuits and their use in Increasing Circuit Robustness. PhD thesis, University of Washington, Seattle, Washington, 1994.

[13] N. Maheshwari and S. S. Sapatnekar. An improved algorithm for minimum-area retiming. In ACM/IEEE Design Automation Conference (DAC), pages 2-7, June 1997.

[14] N. Maheshwari and S. S. Sapatnekar. Minimum area retiming with equivalent initial states. In IEEE/ACM International Conference on Computer-Aided Design (ICCAD), pages 216-219, Nov. 1997.

[15] S. Malik, E. M. Sentovich, R. K. Brayton, and A. Sangiovanni-Vincentelli. Retiming and resynthesis: Optimizing sequential networks with combinational techniques. IEEE Transactions on Computer-Aided Design of Integrated Circuits and Systems, 10(1):74-84, Jan. 1991.

[16] N. Shenoy and R. Rudell. Efficient implementation of retiming. In IEEE/ACM International Conference on ComputerAided Design (ICCAD), pages 226-233, Nov. 1994.

[17] N. V. Shenoy, K. J. Singh, R. K. Brayton, and A. L. Sangiovanni-Vincentelli. On the temporal equivalence of sequential circuits. In ACM/IEEE Design Automation Conference (DAC), pages 405-409, June 1992.

[18] V. Singhal, S. Malik, and R. K. Brayton. The case for retiming with explicit reset circuitry. In IEEE/ACM International Conference on Computer-Aided Design (ICCAD), pages 618-625, Nov. 1996.

[19] H. J. Touati and R. K. Brayton. Computing the initial states of retimed circuits. IEEE Transactions on Computer-Aided Design of Integrated Circuits and Systems, 12(1):157-162, Jan. 1993. 